\title{
Abraham, Ulf \& Brendel-Perpina, Ina (Hrsg.) (2017): Kulturen des Inszenierens in Deutschdidaktik und Deutschunterricht. Stuttgart: Filibach bei Klett
}

\section{Dragan Miladinović}

Wie vollzieht sich sprachliches und literarisches Lernen in der Mediengesellschaft? Welche Kulturen kommen heutzutage in der Mediengesellschaft und in der Deutschdidaktik zum Tragen und inwiefern werden diese inszeniert? Auf diese und weitere im Zusammenhang stehende Fragen geht der vorliegende Sammelband ein und reflektiert die Deutschdidaktik im Hinblick auf die neu entstehenden (und bereits vorhandenen), gesellschaftlich-kulturellen Praxen. Er folgt auf das Symposion Kulturelle Dimensionen sprachlichen Lernens, das 2016 in Ludwigsburg stattfand.

In der Einleitung werden die zwei Schlüsseltermini Inszenierung und Kul$\operatorname{tur}(e n)$, die bereits im Titel des Bandes aufscheinen, durch die Herausgebenden Ulf Abraham und Ina Brendel-Perpina begriffstheoretisch erläutert, nicht zuletzt deshalb, weil sie häufig unscharf und so auch im Alltagsdiskurs Verwendung finden. Die Einleitung dient den folgenden Beiträgen als Basis und bettet den Sammelband kulturwissenschaftlich ein.

Vorweggenommen sei, dass in der Einleitung dem Kulturbegriff mehr Beachtung gegeben wird; der Inszenierungsbegriff wird im Vergleich dazu kürzer abgehandelt. Das Kulturverständnis, das dem Band zugrunde liegt, geht von der „Verschiedenartigkeit und Gleichwertigkeit von Kulturen und kulturellen Ausdrucksformen [aus]“, da dies die Möglichkeit gebe, möglichst viel von dem zu berücksichtigen, was aktuell und auch zukünftig die Sprachund Medienkultur beeinflussen und prägen werde (10). Weiters beruht das Verständnis auf zwei Prämissen: Kultur umfasse „erlerntes Wissen und erlernte Fähig- und Fertigkeiten" und gleichzeitig sei kulturelles Wissen gesellschaftlich geteilt (9). Zusätzlich zu kulturellem Wissen sei aber auch noch kulturelles Können vonnöten, da Kultur „in Praxen des Handelns und Gestaltens, die einerseits voraussetzen, andererseits dazu beitragen, dass etwas gekonnt wird", entstehe (ibid., Herv. i. O.). Der Sammelband setzt sich als eines seiner Ziele, herauszuarbeiten, welche Kulturen sprachlicher Inszenierung in der heutigen Gesellschaft existieren, wie diese in den Unterricht $\mathrm{zu}$ bringen sind und gleichzeitig zu zeigen, was erfahren, gelernt und geübt werden muss, um für die gesellschaftliche Teilhabe notwendige Kompetenzen zu entwickeln (ibid.). In diesem Zusammenhang sei die kulturelle Bildung als erweitertes „Konzept 
ästhetischer Bildung in den explizit sozialen und politischen Dimensionen eines breiten Kulturbegriffs" (11) bedeutsam. Dem Cultural Turn in den Sozial- und Geisteswissenschaften folgend, sind kulturelle Praxen als die Ausbildung und Aushandlung sozialer und kultureller Identität in gemeinsamen Gestaltungsprozessen zu verstehen. Das hebe hervor, dass kulturelle Bildung für die Teilhabe am kulturellen und gesellschaftlichen Leben notwendig sei. Kulturelle Bildung müsse Erfahrungs- und Kommunikationsgelegenheiten bieten, damit leiblich erfahren, spielerisch geübt, künstlerisch produziert und reflektiert werden kann.

Der Begriff der Inszenierung spiele auf drei Ebenen eine Rolle: Zum einen im Sinne des performativen Akts der Aufführung, zum anderen im anthropologischen Sinne des gesellschaftlichen und sozialen In-Szene-Setzens. Das letzte Verständnis bezieht sich auf die Unterrichtsplanung, d.h. auf die didaktisch-methodischen Möglichkeiten wie das Konzipieren, Strukturieren und Bereitstellen von Lernsituationen und -räumen (13).

In insgesamt fünf Themenfeldern werden die unterschiedlichen Kulturen sprachlicher Inszenierung besprochen.

Im ersten Abschnitt drehen sich die Beiträge um Mündlichkeit. Jürgen Belgrad, Corinna Böhme, Sandra Holm und Nicole Schick stellen ein Projekt zur Lese- und Sprachförderung vor, das um das gestische Vorlesen erweitert wurde (25-42). Gestisches Vorlesen macht im Vergleich zum traditionellen Vorlesen aus Zuhörer*innen auch Zuseher*innen, die sich auf die unterstützenden Gesten, Bilder, den Stimm- und Körpereinsatz fokussieren und diese als Verständnishilfe heranziehen können. Hans Lösener und Christiane Saknus führen den Begriff des poetischen Sprachenlernens ein und sehen Inszenierungen im Umgang mit Literatur, d.h. das Vorlesen, szenische Präsentieren oder das Arbeiten mit Standbildern, als bedeutsam, da diese erst die sinnliche Erfahrung ermöglichen und diese sei für das Wahrnehmen, Verstehen und Reflektieren von Literatur vonnöten (43-54). Sie erarbeiten anhand der Analyse eines durchgeführten Unterrichtsprojekts zum Erzählen im Deutsch-als-Zweitsprache-Unterricht vier Thesen poetischen Sprachlernens. Sabine Pfäfflin stellt ein Projekt der Pädagogischen Hochschule Ludwigsburg vor, in dessen Zentrum die Sprechgestaltung und Vertonung von Lyrik steht (55-70).

Der zweite Teil ist der Theatralität und medialer Inszenierung gewidmet. Nina Simon plädiert für eine stärkere „Berücksichtigung poststrukturalistischer und rassismuskritischer Perspektiven " in der universitären Deutschlehrausbildung (73-86). Sie stellt in ihrem, den Kulturbegriff kritisch reflektierenden und dekonstruierenden Beitrag den konzeptionellen und theoretischen Rahmen ihres Promotionsvorhabens vor und deutet die Eignung von theaterpädagogischen Ansätzen zur rassismuskritischen Arbeit in der Lehrausbildung an. Von einer filmischen Inszenierungsform handeln die beiden folgenden Beiträge von Gabriele Paule (87-100) und Petra Anders (101-113). Während Paule darstellt, welchen Mehrwert die Produktion von Buch- und Theatertrailern hat, zeigt Anders das Potential von der Kurzfilmgattung Gedichtfilm auf, das sowohl in deren Produktion als auch Analyse und Erschließung liegt. 
Im dritten Abschnitt finden Beiträge zu Gender-Performances ihren Platz. Stefan Krammer und Julia Malle (117-132) zeigen, dass es für die Vermittlung von Genderkompetenzen in der Schule hilfreich ist, "Geschlecht aufführen zu lassen und dabei auf performative Formen $\mathrm{zu}$ fokussieren, die das Klassenzimmer zur Bühne machen" (128). Diese Art der Auseinandersetzung mit u.a. der Rollenübernahme würde aber die Wahrnehmung, Reflexion und Dekonstruktion von Geschlechter-Inszenierungen bzw. von Geschlechterrollen und -verhältnissen ermöglichen. Wiebke Dannecker erläutert anhand der Arbeit mit dem Roman Boy2Girl von Terence Blacker Inszenierungen von Gender-Rollen sowie das Potenzial von Gender-Performances als Zugang zur literarästhetischen Auseinandersetzung (133-144).

Orte und Räume stehen im Vordergrund des vierten Teils. Hier bieten zunächst Lukas Bannwart und Martin Brändli (147-160) Einblick in ihr Lernangebot „Literatur vor Ort“, bei dem Studierende der PH Luzern „vier Tage literarisch in der Zentralschweiz unterwegs sind." (147) Sie verfolgen dabei einen identitätsorientierten und persönlichkeitsbildenden Ansatz literarischen Lernens und arbeiten heraus, inwiefern die Anwesenheit vor Ort die Rezeption und Produktion von Literatur beeinflusst. Katrin Lehnen und Sara Rezat demonstrieren, wie sich materialgestütztes Schreiben für kulturelles Lernen einbringen kann (161-178). Der Lernraum, der hier betreten wird, ergibt sich u.a. durch die offene Aufgabenkonstruktion, die „einen praktischen Anlass gibt und für den anvisierten Zieltext [...] einen vergleichsweise großen Spielraum der Umsetzung eröffnet" (168) und somit ein Arbeiten ermöglicht, das über den Kulturraum Schule hinausgeht. Um den virtuellen Raum geht es im Beitrag von Christine Gehl-Trepkau (179-191), die WebQuests als Möglichkeit sieht, Lernende im digitalen Zeitalter für klassische Inhalte $\mathrm{zu}$ motivieren sowie „ihre sprachlichen, medialen und/oder literarischen Kompetenzen“ (179) zu erweitern.

Im letzten Abschnitt des Bandes wird die Produktionsorientierung als Inszenierung aufgefasst. Uta Hauck-Thum (195-208) nutzt in ihrem Beitrag Adaptable Books, d.h. sogenannte enhanced eBooks, die in einer App bearbeitet werden können, als Inszenierungsräume für aktive und eigenverantwortliche sowie an die persönlichen Bedürfnisse angepasste Mitgestaltung von Büchern.

Hartmut Vollmer (209-222) bezieht sich in seinem Beitrag auf handlungs- und produktionsorientierten Literaturunterricht und plädiert für die Herausbildung der Imaginationsfähigkeit, beispielsweise durch pantomimische Textinterpretation. Diese ermögliche „eine sinnliche Intensivierung der Texterschließung, ein sinnliches Verstehen von Wörtern, Begriffen und Texten." (220) Schließlich arbeiten Petra Anders und Judith Riegert (223-235) das Potenzial von Handlungsund Produktionsorientierung für inklusiven Literaturunterricht heraus.

Die Beiträge bieten zahlreiche Einblicke in vielfältige Projekte und theoretische Überlegungen, die sich mit diesen unterschiedlichen Inszenierungsformen beschäftigen und die den Deutschunterricht auf ihre Weise bereichern und erweitern: von gestischem Vorlesen als Sprach- und Leseförderungsunterstützung, über theaterpädagogische Auseinandersetzung mit Rassismuskritik in der 
Deutschlehrendenbildung, über Geschlechterinszenierungen und -reflexion, bis hin zum Potential von handlungs- und produktionsorientierten Verfahren. Die aktive Inbezugnahme des Körpers, sei es mit Standbildern, mit kurzen szenischen Darstellungen bzw. Interpretationen oder dem gestischen Gestalten von Texten, sowie das ästhetische Lernen, die von Beiträgen eingefordert werden, sind im Sinne einer performativen, körperbetonten Didaktik (vgl. Schewe 2013) zu begrüßen. Die gelungene Mischung aus Empirischem und Didaktischem macht den Sammelband besonders wertvoll und er eignet sich aus diesem Grund und aufgrund seiner vorgestellten Vielfalt für ein breites Publikum, das sich mit dem Inszenierungscharakter von Kulturen beschäftigen möchte.

\section{Bibliografie}

Schewe, Manfred (2013): Taking Stock and Looking Ahead: Drama Pedagogy as a Gateway to a Performative Teaching and Learning Culture. In Scenario VII/1, 5-23. http://research.ucc.ie/scenario/2013/01/Schewe/02/en [letzter Zugriff: 10.12.2018] 